\title{
Synthetic Aperture Guided Wave Imaging using a Mobile Sensor Platform
}

\author{
Gordon Dobie, Walter Galbraith, Charles MacLeod, Rahul Summan and Gareth Pierce \\ Centre for Ultrasonic Engineering \\ Department of Electronic and Electrical Engineering \\ University of Strathclyde \\ 204 George Street, Glasgow, G1 1XW, UK \\ web: www.cue.ac.uk
}

\begin{abstract}
Miniature robotic vehicles are receiving increasing attention for use in Non-Destructive Evaluation (NDE) due to their attractiveness in terms of cost, safety and their accessibility to areas where manual inspection is not practical. Under ideal conditions, ultrasonic guided waves can propagate for significant distances across structures (10's meters) enabling rapid inspection of large areas. Furthermore guided waves can be generated by non-contact transducers which offers the possibility of couplant-free ultrasonic inspection. Combining non-contact guided wave transducers with mobile sensor platforms offers a unique opportunity for rapid reconfigurable inspection of large structures. This paper presents a two stage strategy for the inspection of structures made up of a series of plates sections, such as gas storage tanks. The first stage identifies the robot's position relative to the plate section on which it sits. The second stage inspects the plate using a synthetic array created from a series of measurements take across the width of the plate section.
\end{abstract}

\section{INTRODUCTION}

Miniature Remote Sensing Agents (RSAs) are well suited for the inspection of many structures and installations. Onboard power and wireless communications obviates the need for umbilical cabling, maximising access and mobility. Their small size and magnetic traction makes them particularly useful for inspecting areas with limited access allowing them to crawl over the surface of a structure, along pipes, and into small spaces. Moreover, the concept can be extended to a fleet of heterogeneous robots which improves the functionality, reliability and flexibility of the system as a whole. This paper considers a specific type of miniature robot, deploying a non-contact ultrasonic inspection sensor. Unlike conventional ultrasound sensors which generate and measure ultrasonic bulk waves transmitted into the depth of the sample, this ultrasonic payload makes use of Lamb waves, a type of guided ultrasonic wave [1] where the plate-like structure forms a wave-guide and the wave travels across the structure. Guided waves offer significant potential for the following reasons:

1) Lamb waves can be generated and measured with noncontact air-coupled angled transducers [2].

2) Lamb waves can propagate over long distances (tens of meters) increasing the efficiency of inspections over those based on spot measurements.

This paper considers the use of a single robot mounted sensor that generates Lamb waves and constructs an image from measured echos. It begins by briefly describing the inspection system, before presenting two algorithms that can be combined to form a reconfigurable strategy for scanning structures made up of a series of plates or panels. The first algorithm identifies the position of a robot relative to a plate sections. The algorithm then uses the Synthetic Aperture Focusing Technique (SAFT) to image the plate section. The authors show that is possible to use reflections from the plate edge to enhance the robot's positional accuracy, improving SAFT results.

\section{SYSTEM OVERVIEW}

The robotic sensor platform is a differentially driven two wheel vehicle with a passive castor. The current prototype is approximately $175 \times 124 \times 85$ and can include magnetic wheels for traction on inclined ferromagnetic surfaces. A photograph is shown in Figure 1. A pair of piezoelectric air coupled transducers were mounted on the front of the robot inclined at an appropriate angle to the test specimen to generate the zeroth order anti-symmetric $\left(A_{0}\right)$ Lamb wave in plate-like structures. $A_{0}$ Lamb waves are typically generated in samples where the plate thickness is less then the wavelength, for example at $600 \mathrm{kHz}$ the thickness should be less than $3.3 \mathrm{~mm}$. It is important to note that $A_{0}$ Lamb wave mode is dispersive, so the phase velocity is dependent on the sample's material properties, its geometry and the frequency of operation [1]. Discontinuities in the structure cause reflections of the Lamb wave signal and it is by this mechanism that defects are detected. It should be noted that Lamb wave propagation is a function of the specimen's elastic properties and that factors, such as structural loading, will effect Lamb wave propagation. However, these changes are typically negligible when compared to the effect of defects. Previous work by the authors [3] evaluates the suitability of air-coupled piezoelectric transducers to different sample geometries and the feasibility of synthetic aperture imaging using the transducers in this configuration [4].

\section{DESCRIPTION OF THE INSPECTION PROBLEM}

This work concerns itself with structures that comprise of a series of plate sections, often welded or riveted onto a supporting frame, such as gas or oil storage tanks or 


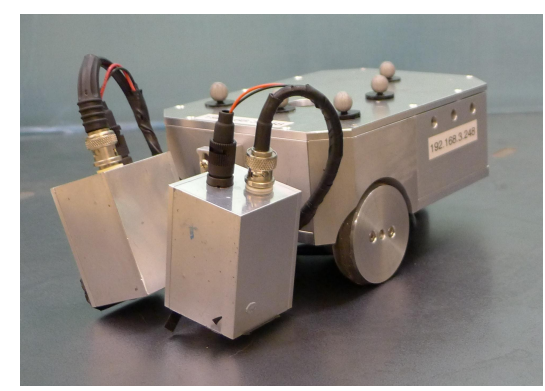

Fig. 1: Ultrasonic inspection robot showing air-coupled ultrasonic payload.

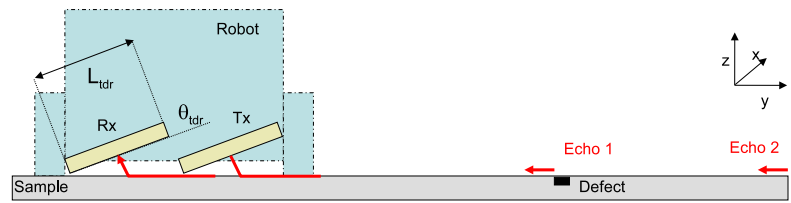

Fig. 2: Schematic of robot mounted air-coupled sensors configured for pulse-echo inspection.

aircraft fuselage and wing sections. The sharp discontinuities between panels can reflect a large portion of the guided wave, so the effective inspection of such a structure should consider the panel layout, ideally inspecting plate sections individually. In addition to this, robot positional error can have a significant effect on image reconstruction [4], where the error can easily exceed the wavelength of the ultrasonic signal used for inspection. If the geometry of the plate is known it can be used to correct for errors in the robot's position. In many cases the layout of the plate sections on a structure is unavailable or their retrieval and conversion into an appropriate form is not viable. It would be more useful if the robot could extract this information from the structure with little or no a priori information. Section V demonstrates an algorithm to extract the current plate boundary from ultrasonic measurements. Section VI then demonstrates an algorithm that can correct for robot positional uncertainty using information about the plate boundary. Before discussing these algorithms, the extract of range information from the ultrasonic signals should be discussed.

\section{EXTRACTION OF RANGE INFORMATION FROM GUIDED WAVE REFLECTIONS}

At frequency thickness products below $2 \mathrm{MHz}-\mathrm{mm}$ the first order antisymmetric Lamb wave is highly dispersive [1]. This makes the propagation velocity frequency dependant which leads to an elongation of transmitted signals which results in a reduction in imaging resolution. Non-contact air-coupled inspection is typically performed at relatively low operating frequencies (in this case $600 \mathrm{kHz}$ ) to minimise attenuation loss in the air channel [5], so often has to operate in this dispersive region. In this work a numerical dispersion compensation technique by Sicard et al [6] was used. It processes the individual A-Scans in the frequency domain, using the theoretical Lamb wave phase propagation velocity for each frequency component.

\section{EXTRACTION OF PLATE BOUNDARIES}

The algorithm extracts plate boundaries from a series of ultrasonic ranging measurements. The only assumption is that the plate is rectangular - there is no a priori knowledge of the dimensions or the robot's pose relative to the plate (including rotation). The robot performs a radial B-scan from a random location. An Finite Difference simulated result [7] of the sample shown in Figure 3a is shown in Figure 3b. In this case 72 UT measurements were collected at $5^{\circ}$ increments.

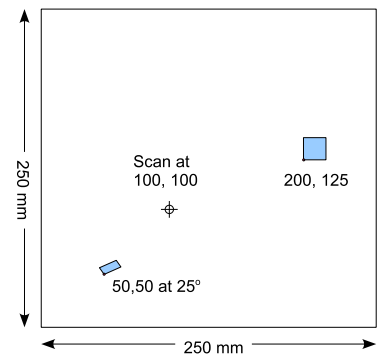

(a)

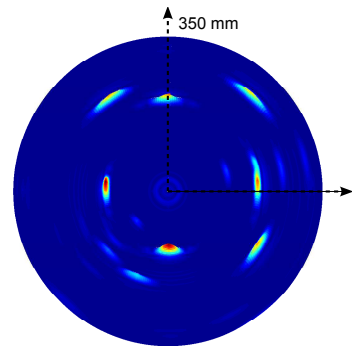

(b)
Fig. 3: (a) Schematic of a $250 \times 250 \times 1 \mathrm{~mm}$ plate sample with two artificial defects. (b) Simulation of a polar B-scan of the $250 \times 250 \times 1 \mathrm{~mm}$ plate section shown in (a).

Reflections from the plate edges and corners are clearly visible. There are also faint reflections from the artificial defects. The individual A-scans are converted from the temporal domain to the spatial domain using the algorithm discussed in Section IV. All echos that were $6 \mathrm{~dB}$ above the noise floor are extracted and converted to Cartesian coordinates. The exact location of the echo was taken as a fixed distance in front of the peak of the reflection, this proved to be more reliable than using a threshold. The process is then repeated at a second random location to collect a second dataset. The plate boundary was assumed to be rectangular and was therefore defined the five variables: the location of the bottom left corner $(\mathrm{x}, \mathrm{y})$, the width ' $\mathrm{W}$ ', the length ' $\mathrm{L}$ ' and the angle of rotation from the normal ' $\theta$ '. The distance between each point and this rectangle was calculated as the shortest distance between the point and the four line segments. The quality of a fit was defined as the mean squared error (MSE) of these distances. Standard optimisation techniques were be applied to solve for the 'best-fit' rectangle. In this case Matlab's 'fminunc' function was used [8].

In order to reduce the algorithms sensitivity to spurious reflections from defects, a threshold was set for a maximum 'average point deviation' from the rectangle (i.e. $5 \mathrm{~mm}$ ). If the optimisation solution fell above this threshold the worst offending point was removed. This process was repeated until the error fell below the maximum deviation threshold. An example fit is shown in Figure 4. In this case seven points were removed before a satisfactory fit was achieved (removed 
points are shown as crosses). The resulting boundary estimate error was less than $5 \mathrm{~mm}$ for $x_{0}, y_{0}, \mathrm{~L}$ and $\mathrm{W}$ and $0.6^{\circ}$ for $\theta$.

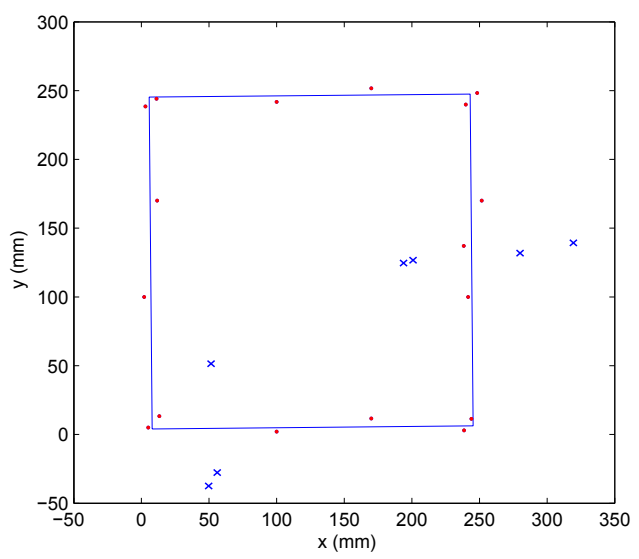

Fig. 4: Resulting plate boundaries in the presence of spurious reflections from defects.

\section{The Synthetic Aperture Focusing Technique}

Once the plate boundary's have been estimated, the plate was imaged using the Synthetic Aperture Focusing Technique (SAFT). In most cases the robot drives across the centre of the plate twice, once imaging the top half of the plate and once the bottom half. SAFT [9] is a back propagation technique that enhances B-scans by superimposing reflections generated and measured by a single transducer at multiple locations, typically linearly spaced along a single axis. Multiple receiver locations observe the same reflection, but in each case the reflection is time shifted due to a difference in the distances between the reflector and the individual transducer. The SAFT algorithm phase shifts the receiver time histories to compensate for the non-uniform propagation distance and therefore aligns the echo's from each transducer location. SAFT is implemented by scanning the field of view and at each image location (pixel), summing the response from each transducer with the appropriate phase shift.

There are a number of issues that occur when applying SAFT to a mobile robotic vehicle with air-coupled guided wave transducers:

1) Positional error in transducer placement introduces errors in phase compensation. At $600 \mathrm{kHz}-\mathrm{mm}$ the phase wavelength is $3.7 \mathrm{~mm}$. Since positional accuracy must be significantly less than half a wavelength, this puts a tight requirement of robot positional accuracy. Furthermore if the transducer's are not at the robot's centre of rotation, any angular error leads to translation of transducer positions.

2) Given that the insertion loss experienced with aircoupled transducers is typically $80 \mathrm{~dB}$, ensuring adequate SNR is a challenge. Relatively large transducers (i.e. $30 \times 30 \mathrm{~mm}$ ) are typically used to achieve resonable SNR. However, when inspecting $1 \mathrm{~mm}$ aluminium a 30 mm square transducer produces an aperture of $8 \lambda$. This means that the transducer beam cannot be reasonably approximated as a radial source. When the transducers act as a planer source, beam steering is no longer practical, so phase compensation is not effective. Furthermore the system is sensitive to angular rotation of the transducer, which now changes the distance from a reflector to the closest part of the transducer face.

3) The large difference in dynamic range between the transmitter and receiver (typically $80 \mathrm{~dB}$ ) means that air-coupled systems require split transmit/receive transducers. This means that the transmitter and receiver must be at different locations which complicates SAFT.

4) Since the propagation velocity in air is only about $15 \%$ of the phase velocity in aluminium, small changes in the length of the air channel lead to significant phase delays, which if not kept constant will distort the image.

\section{EXPERIMENTAL INSPECTION}

Figure 6a applies conventional SAFT to the raw data set shown in Figure 5. The dataset collected by the robotic vehicle shown in Figure 1. The sample was a $1.5 \mathrm{~mm}$ aluminium plate with a $30 \times 10 \mathrm{~mm}$ artificial defect at $50 \%$ depth. Applying conventional SAFT produced Figure 6a. Due to the planar nature of the sources the signals, phase compensation broke down. In this case it was preferable to use the magnitude of signals that had been transformed using the Hilbert transform when performing SAFT, the result of which is shown in Figure $6 b$.

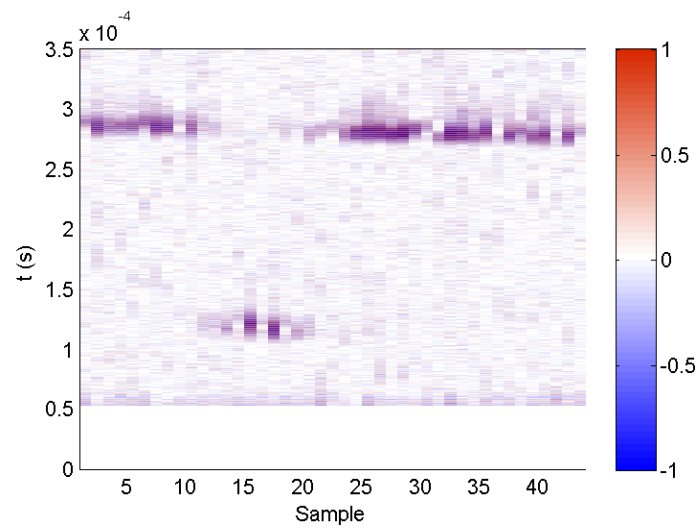

Fig. 5: Ultrasonic data set collect by the miniature robotic vehicle shown in Figure 1.

\section{BACK EDGE SAFT CORRECTION}

If the plate edges can be assumed to be flat, it is possible to use information from the back wall reflections to refine the robot's positional estimate. This works best if the transducer can be assumed to be a radial source, so that the distance to the back wall is not a function of the XY angle of the transducer relative to the plate edge. Furthermore the transducer should be mounted at the axis of rotation of the robotic vehicle, so that if the robot accidentally turns slightly, there is no $\mathrm{X}, \mathrm{Y}$ 


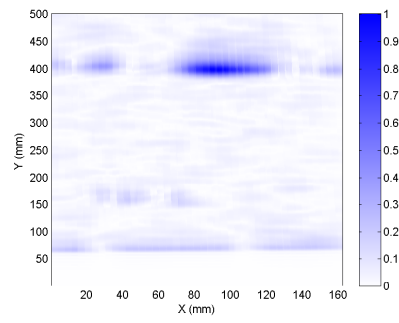

(a)

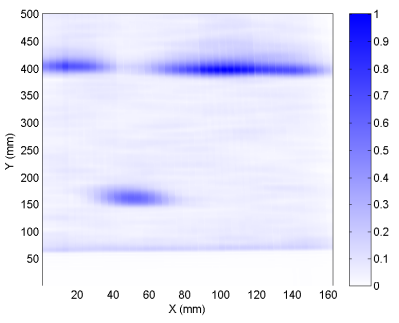

(b)
Fig. 6: (a) SAFT processing of data set shown in Figure 5. (b) SAFT processing of data set shown in Figure 5 ignoring phase information.

change in the centre of the split transducer. The authors have not yet created a robot to meet the second requirement, so the results from Finite Difference simulations were used to demonstrate the algorithm. The transducer's were $5 \mathrm{~mm} \times 15$ $\mathrm{mm}$, and so produced a divergent beam that could be assumed to be radial. The sample was $125 \times 125 \times 1 \mathrm{~mm}$, with a 3 $\mathrm{mm}$ square, $1 \mathrm{~mm}$ deep artificial defect located at $(72.5,70$ $\mathrm{mm})$. Noise was added to each successive transducer position, so that across the $65 \mathrm{~mm}$ wide scan path (46 measurements), there was a cumulative error of $2 \mathrm{~mm}$ in $\mathrm{X}$ and $3 \mathrm{~mm}$ in $\mathrm{Y}$. The result of SAFT processing is shown in Figure 7a where there is a clear degradation in the back edge reflection.

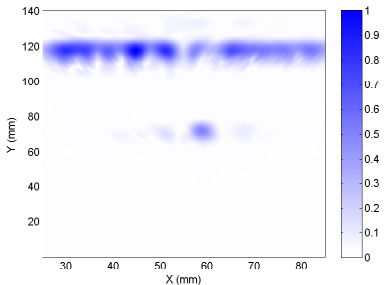

(a)

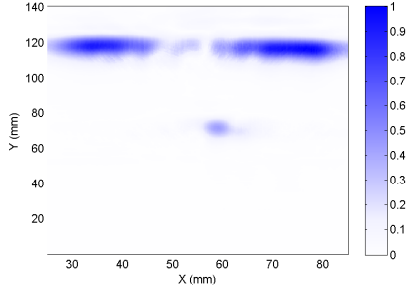

(b)
Fig. 7: (a) SAFT processing of simulated data with transducer misplacement. (b) Refinement of (a) where phase errors were compensated for using information from the back edge echo.

The back edge echo was extracted from each raw signal and the phase error between successive measurements was calculated. These erroneous phase shifts were then nullified and the data reprocessed using SAFT. The resulting image is shown in Figure $7 \mathrm{~b}$. Note both the improvement in the back wall and the image of the defect.

\section{CONCLUSION}

This paper has shown a two stage algorithm that can be used to inspect structures made of multiple plate sections (such as gas storage tanks) using a miniature robotic vehicle with guided wave transducers. In this work air-coupled guided waves were used, but the algorithms are applicable to other types of sensor such as EMATs.

The first stage of the algorithm estimated the robots position relative to a particular plate section by fitting a rectangle to plate boundary reflections. It was shown to be robust, even in the presence of additional reflectors. Once the plate boundary was established SAFT was used to image the plate section. The authors discovered a number of complications when applying SAFT to data collected by an air-coupled guided wave miniature robotic vehicle. Most notably that large aperture transducers did not act as a radial source, so that SAFT phase compensation was not effective and that even if a radial source could be achieved. Also that slight robot positional uncertainty lead to a break down in phase compensation.

The authors demonstrated that reflections from the back edge of the plate could be used to enhance the robot's positional estimation and that if a non-planar source was used, such an estimation could be used to reduce errors in phase compensation, enhancing SAFT imaging.

\section{ACKNOWLEDGMENT}

This research received funding from the Engineering and Physical Sciences Research Council (EPSRC) and forms part of the core research programme within the UK Research Centre for NDE (RCNDE).

\section{REFERENCES}

[1] I. A. Viktorov. Rayleigh and Lamb waves: Physical theory and applications. Plenum Press, 1967.

[2] R. Farlow and G. Hayward. An automated ultrasonic NDT scanner employing advanced air-coupled 1-3 connectivity composite transducers. Insight, 38(1):41-50, 1996.

[3] G. Dobie, R. Summan, S. Pierce, W. Galbraith, and G. Hayward. A noncontact ultrasonic platform for structural inspection. Sensors Journal, IEEE, (99):1-1.

[4] Gordon Dobie. Ultrasonic Sensor Platforms for Non-Destructive Evaluation. $\mathrm{PhD}$ thesis, University of Strathclyde, 2010.

[5] R. Farlow and G. Hayward. Real-time ultrasonic techniques suitable for implementing non-contact NDT systems employing piezoceramic composite transducers. Insight, 36(12):926-935, 1994.

[6] R. Sicard, J. Goyette, and D. Zellouf. A numerical dispersion compensation technique for time recompression of lamb wave signals. Ultrasonics, 40(1-8):727-732, 2002.

[7] Gordon Dobie, Andrew Spencer, Kenneth Burnham, S. Gareth Pierce, Keith Worden, Walter Galbraith, and Gordon Hayward. Simulation of ultrasonic lamb wave generation, propagation and detection for a reconfigurable air coupled scanner. Ultrasonics, 51(3):258 - 269, 2011.

[8] MathWorks. Matlab. www.mathworks.co.uk, Accessed Sept 2009.

[9] K. Nagai. A new synthetic-aperture focusing method for ultrasonic Bscan imaging by the Fourier transform. IEEE Transactions on Sonics and Ultrasonics, 32(4):531-536, 1985. 